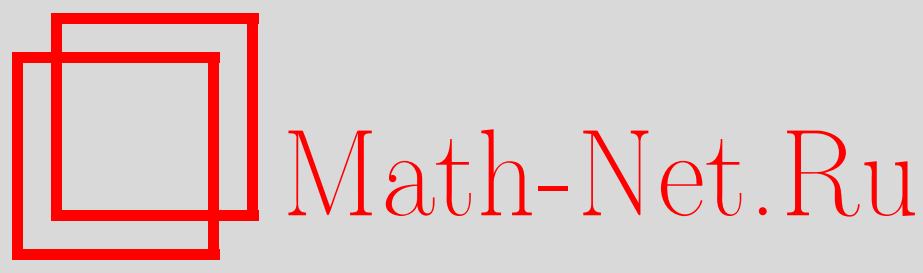

Р. Джакив, Майорановские фермионы и их алгебры Клиффорда со связями, ТМФ, 2014, том 181, номер 1, 39-44

DOI: https://doi.org/10.4213/tmf8701

Использование Общероссийского математического портала Math-Net.Ru подразумевает, что вы прочитали и согласны с пользовательским соглашением http://www.mathnet.ru/rus/agreement

Параметры загрузки:

IP: 54.92 .164 .108

26 апреля 2023 г., 15:48:39

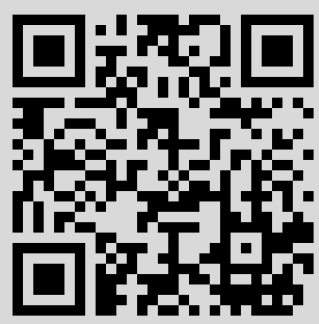




\title{
ФИЗИКА
}

Том 181, № 1

октябрь, 2014

(C) 2014 г.

\author{
P. Джакив*
}

\section{МАЙОРАНОВСКИЕ ФЕРМИОНЫ И ИХ АЛГЕБРЫ КЛИФФОРДА СО СВЯЗЯМИ}

Приведен пример, сводящий воедино математику (алгебры Клиффорда со связями) и физику (майорановские фермионы).

Ключевые слова: майорановские фермионы, алгебры Клиффорда, топологические возбуждения.

DOI: $10.4213 / \operatorname{tmf} 8701$

Посвящается Людвигу Фаддееву в связи с его восъмидесятилетием. Людвиг оличетворяет собой идеал математического физика с его весомым вкладом в математику (алгебраические свойства интегрируемых систем) и в физику (квантовая теория поля).

В квантовой теории поля комплекснозначные квантовые поля описывают заряженные частицы и античастицы. Нейтрально заряженные возбуждения, которые задаются самосопряженными выражениями (частицы совпадают с античастицами), задаются вещественнозначными полями. Реальным примером может служить или нейтральный пион с нулевым спином, или фотон, который также нейтрален, но имеет спин 1, или (гипотетический) гравитон со спином 2. Все эти частицы являются бозонами. Но что можно сказать о самосопряженных фермионах? Надо признать, что пока нет экспериментальных данных, свидетельствующих в пользу их существования. Тем не менее в современных теориях такие частицы используются для объяснения последних данных, полученных в нейтринной физике. Также и суперсимметричные партнеры самосопряженных бозонов должны быть самосопряженными фермионами.

Уравнение Майораны описывает самосопряженные фермионы в терминах вещественнозначного уравнения Дирака. Стандартное комплекснозначное уравнение Дирака имеет вид

$$
(\boldsymbol{\alpha} \cdot \mathbf{p}+\beta m) \Psi=i \frac{\partial}{\partial t} \Psi
$$

${ }^{*}$ Center for Theoretical Physics, Massachusetts Institute of Technology, Cambridge, MA, USA. E-mail: jackiw@mit.edu 
где $\Psi$ - четырехкомпонентная комплексная функция (описывающая заряженные возбуждения), $\boldsymbol{\alpha}, \boldsymbol{\beta}$ - четыре “дираковские" числовые $(4 \times 4)$-матрицы, $\mathbf{p}=\frac{1}{i} \boldsymbol{\nabla}, m-$ массовый параметр. Матрицы Дирака гарантируют, что итерация дает

$$
-\frac{\partial^{2}}{\partial t^{2}} \Psi=-\nabla^{2} \Psi+m^{2} \Psi
$$

Уравнение Дирака $= \pm \sqrt{\text { волновое уравнение массивного поля. }}$

Уравнение Дирака производит "разложение” волнового уравнения массивного поля, извлекая из него “квадратный корень" в матричных терминах. Но так как квадратные корни могут быть разных знаков, комплексное уравнение Дирака описывает фермионные частицы одновременно с фермионными античастицами. Ясно, что можно произвести аналогичное разложение, в котором частицы будут отождествлены с античастицами, т. е. с вещественнозначной волновой функцией $\Psi$. Анализируя приведенное выше уравнение Дирака, можно заключить, что функция $\Psi$ может быть вещественной при мнимом $\beta$, а поскольку $\mathbf{p}=-i \boldsymbol{\nabla}$, то $\boldsymbol{\alpha}$ должно быть вещественным. Такой выбор матриц Дирака задает представление Майораны.

Конкретный пример представления Майораны для таких матриц дается выражениями

$$
\begin{gathered}
\alpha_{\mathrm{M}}^{1}=\left(\begin{array}{cc}
0 & \sigma^{1} \\
\sigma^{1} & 0
\end{array}\right), \quad \alpha_{\mathrm{M}}^{2}=\left(\begin{array}{cc}
I & 0 \\
0 & -I
\end{array}\right), \quad \alpha_{\mathrm{M}}^{3}=\left(\begin{array}{cc}
0 & \sigma^{3} \\
\sigma^{3} & 0
\end{array}\right), \\
\beta_{\mathrm{M}}=\left(\begin{array}{cc}
0 & \sigma^{2} \\
\sigma^{2} & 0
\end{array}\right), \quad \Psi_{\mathrm{M}}^{*}=\Psi_{\mathrm{M}}, \\
\boldsymbol{\alpha}_{\mathrm{M}}^{*}=\boldsymbol{\alpha}_{\mathrm{M}}, \quad \beta_{\mathrm{M}}^{*}=-\beta_{\mathrm{M}} .
\end{gathered}
$$

Но так как физическое содержание инвариантно относительно преобразований сопряжения, приведенное выше условие вещественности можно заменить на условие вещественности с точностью до сопряжения, задаваемого матрицей сопряжения $C$ :

$$
C \boldsymbol{\alpha}^{*} C^{-1}=\boldsymbol{\alpha}, \quad C \beta^{*} C^{-1}=-\beta, \quad C \Psi^{*}=\Psi .
$$

Например, если использовать представление Вейля

$$
\boldsymbol{\alpha}=\left(\begin{array}{cc}
\boldsymbol{\sigma} & 0 \\
0 & -\boldsymbol{\sigma}
\end{array}\right), \quad \beta=\left(\begin{array}{cc}
0 & I \\
I & 0
\end{array}\right),
$$

то матрица сопряжения примет вид ${ }^{1)}$

$$
C=\left(\begin{array}{cc}
0 & -i \sigma^{2} \\
i \sigma^{2} & 0
\end{array}\right) .
$$

Таким образом, уравнение Майораны сохраняет вид уравнения Дирака (Вейля), но с наложенным условием сопряжения $C \Psi^{*}=\Psi$ :

$$
\begin{gathered}
\Psi=\left(\begin{array}{c}
\psi \\
\chi
\end{array}\right), \quad\left(\begin{array}{cc}
\boldsymbol{\sigma} \cdot \boldsymbol{p} & m \\
m & -\boldsymbol{\sigma} \cdot \boldsymbol{p}
\end{array}\right)\left(\begin{array}{l}
\psi \\
\chi
\end{array}\right)=i \frac{\partial}{\partial t}\left(\begin{array}{l}
\psi \\
\chi
\end{array}\right), \\
C \Psi^{*}=\Psi \quad \Rightarrow \quad \chi=i \sigma^{2} \psi^{*} .
\end{gathered}
$$

1)В представлении Майораны $C=I$. 
Условие связи приводит к двухкомпонентному майорановскому $(2 \times 2)$-матричному уравнению

$$
\boldsymbol{\sigma} \cdot \boldsymbol{p} \psi+i \sigma^{2} m \psi^{*}=i \frac{\partial}{\partial t} \psi
$$

Заметим, что $\psi$ смешивается с $\psi^{*}$; майорановский массовый член не сохраняет никакого квантового числа, и нет различия между частицами и античастицами ввиду отсутствия квантовых чисел, различающих эти два сорта частиц, т. е. частица является своей античастицей.

Полевое разложение для заряженного дираковского поля имеет вид

$$
\Psi=\sum_{E>0}\left(a_{E} e^{-i E t} \Psi_{E}+b_{E}^{\dagger} e^{i E t} C \Psi_{E}^{*}\right)
$$

где $a$ и $b^{\dagger}$ - операторы уничтожения частиц и рождения античастиц соответственно. Для майорановского поля получим

$$
\Psi=\sum_{E>0}\left(a_{E} e^{-i E t} \Psi_{E}+a_{E}^{\dagger} e^{i E t} C \Psi_{E}^{*}\right) .
$$

Операторы античастиц $\left(b, b^{\dagger}\right)$ при этом исчезают.

Замечательное обстоятельство состоит в том, что теоретики в области конденсированных состояний материи столкнулись с точно таким же уравнением при описании контакта сверхпроводника с топологическим изолятором. Соответствующая двумерная гамильтонова плотность задает уравнения движения двухкомпонентного вектора $\psi=\left(\begin{array}{c}\psi_{1} \\ \psi_{2}\end{array}\right)$ и имеет вид

$$
H=\psi^{*}\left(\boldsymbol{\sigma} \cdot \frac{1}{i} \boldsymbol{\nabla}-\mu\right) \psi+\frac{1}{2}\left(\triangle \psi^{*} i \sigma^{2} \psi^{*}+\text { э.c. }\right),
$$

где $\boldsymbol{\sigma}=\left(\sigma^{1}, \sigma^{2}\right), \mu$ - химический потенциал, а $\triangle$ - параметр порядка, который может быть или постоянной величиной $\triangle=\triangle_{0}=m$, или задаваться вихревым профилем, $\triangle(\mathbf{r})=v(r) e^{i \theta}, v(0)=0, v(\infty)=\triangle_{0}$.

Уравнение движения имеет вид

$$
i \partial_{t} \psi=(\boldsymbol{\sigma} \cdot \mathbf{p}-\mu) \psi+\triangle i \sigma^{2} \psi^{*} .
$$

В отсутствие $\mu$ и при постоянном параметре $\triangle$ эта система оказывается $(2+1)$-мерной версией $(3+1)$-мерного двухкомпонентного уравнения Майораны! Она описывает нейтральные фермионы спина $1 / 2$ с майорановской массой $|\triangle|$.

В присутствии единственного вихревого параметра порядка $\Delta(\mathbf{r})=v(r) e^{i \theta}$ имеет место (статическая) изолированная мода с нулевой энергией [1], [2]

$$
\psi_{0}=\#\left(\begin{array}{c}
J_{0}(\mu r) e^{-i \pi / 4-V(r)} \\
J_{1}(\mu r) e^{i(\theta+\pi / 4)-V(r)}
\end{array}\right),
$$

где \# - вещественная постоянная, $V^{\prime}(r)=v(r)$. Полевое разложение для поля Майораны при этом имеет вид

$$
\Psi=\underset{E \neq 0 \text { моды }}{\cdots \ldots \ldots \ldots+a \psi_{0},}
$$


где оператор нулевой моды $a$ удовлетворяет уравнению

$$
\left\{a, a^{\dagger}\right\}=1, \quad a^{\dagger}=a \quad \Rightarrow \quad a^{2}=\frac{1}{2} .
$$

Как реализовать действие $a$ на состояниях? Имеются две возможности [3].

1. Две одномерные реализации. Выберем в качестве вакуумного состояния собственное состояние $а$ с возможными собственными значениями $\pm 1 / \sqrt{2}$ :

$$
a|0 \pm\rangle= \pm \frac{1}{\sqrt{2}}|0 \pm\rangle
$$

Имеются два вакуумных состояния $|0+\rangle$ и $|0-\rangle$. Две башни состояний строятся с помощью многократного применения операторов $a_{E}^{\dagger}$. При этом нет операторов, связывающих эти две башни состояний. Фермионная четность нарушена, так как $a$ - фермионный оператор. Как и в случае спонтанного нарушения симметрии, надо выбрать вакуумное состояние $|0+\rangle$ или $|0-\rangle$, и эти два вакуумных состояния не связаны никаким туннелированием.

2. Одна двумерная реализация. Вакуум при этом двукратно вырожден, $|1\rangle,|2\rangle$, и оператор а переводит эти два состояния друг в друга:

$$
a|1\rangle=\frac{1}{\sqrt{2}}|2\rangle, \quad a|2\rangle=\frac{1}{\sqrt{2}}|1\rangle .
$$

Две башни состояний строятся путем многократного применения оператора $a_{E}^{\dagger}$. Действие оператора $а$ переводит башни состояний друг в друга. Фермионная четность сохраняется.

Естественно предположить, что фермионная четность сохраняется. Поэтому будем предполагать, что выполняется вторая возможность, которая также подтверждается рассмотрением случая пары вихрь-антивихрь, разнесенных на значительное расстояние.

Отметим, что условие нарушения фермионной четности 1 встречается в математической физике. Обозначим через $\mathcal{L}$ лагранжеву плотность для системы "скалярные кинки + фермионы":

$$
\mathcal{L}=\frac{1}{2} \partial_{\mu} \Phi \partial^{\mu} \Phi+\frac{\mu^{2}}{2} \Phi^{2}-\frac{\lambda^{2}}{8} \Phi^{4}+i \bar{\Psi} \gamma^{\mu} \partial_{\mu} \Psi-g \Phi \bar{\Psi} \Psi
$$

$\mathcal{L}$ проявляет свойства суперсимметрии для $g=\lambda, \Psi$.

$\mathrm{C}$ помощью условия аномалии центрального заряда в алгебре суперсимметрии можно доказать, что фермионная четность может и отсутствовать [4]. Остается открытым вопрос, можно ли каким-либо образом реализовать эту экзотическую возможность в теории конденсированного состояния или в какой-либо физически значимой теории (некоторые рассуждения на эту тему см. в работе [5]).

Сколько состояний $\mathcal{N}$ необходимо для представления $N$ вихрей (в виде, сохраняющем фермионную четность)? При $N=1$ используются два состояния: $\mathcal{N}=2$. При $N=2$ имеются эрмитовы операторы $a_{1}$ и $a_{2}$ с $a_{1}^{2}=a_{2}^{2}=1 / 2$ и $a_{1} a_{2}+a_{2} a_{1}=0$. Можно реализовать их действие на двух состояниях, уже присутствующих при $N=1$ :

$$
\begin{array}{ll}
a_{1}|1\rangle=\frac{1}{\sqrt{2}}|2\rangle, & a_{1}|2\rangle=\frac{1}{\sqrt{2}}|1\rangle, \\
a_{2}|1\rangle=\frac{i}{\sqrt{2}}|2\rangle, & a_{2}|2\rangle=-\frac{i}{\sqrt{2}}|1\rangle .
\end{array}
$$


Эти формулы записываются явно, если задать состояния с помощью декартовых 2-векторов, а операторы $a_{i}-$ с помощью матриц Паули:

$$
\begin{aligned}
|1\rangle & \sim\left(\begin{array}{l}
1 \\
0
\end{array}\right), & |2\rangle & \sim\left(\begin{array}{l}
0 \\
1
\end{array}\right), \\
a_{1} & =\frac{\sigma^{1}}{\sqrt{2}}, & a_{2} & =\frac{\sigma^{2}}{\sqrt{2}} .
\end{aligned}
$$

Эти результаты подтверждают формулы $\mathcal{N}=e^{N / 2}$ для четных $N$ и $\mathcal{N}=e^{(N+1) / 2}$ для нечетных $N$.

При $N=3$ имеем операторы трех мод: $a_{i}, i=1,2,3$. Применить три матрицы Паули для того, чтобы представить их в нужном виде, не удается. В частности, нельзя просто положить $a_{3}=\sigma^{3} / \sqrt{2}$, так как матрица $\sigma^{3}$ имеет диагональный вид при действии на декартовы состояния, определенные выше, что должно приводить к нарушению фермионной четности. Поэтому в случае $N=3$ приходится использовать матрицы Дирака размера $4 \times 4$ и декартовы 4-векторы. Для $\mathcal{N}=4$ имеем следующие состояния и операторы:

$$
\begin{gathered}
|1\rangle \sim\left(\begin{array}{l}
1 \\
0 \\
0 \\
0
\end{array}\right), \quad|2\rangle \sim\left(\begin{array}{l}
0 \\
1 \\
0 \\
0
\end{array}\right), \quad|3\rangle \sim\left(\begin{array}{l}
0 \\
0 \\
1 \\
0
\end{array}\right), \quad|4\rangle \sim\left(\begin{array}{l}
0 \\
0 \\
0 \\
1
\end{array}\right), \\
\alpha=\left(\begin{array}{cc}
0 & i \boldsymbol{\sigma} \\
-i \boldsymbol{\sigma} & 0
\end{array}\right), \quad \beta=\left(\begin{array}{ll}
0 & I \\
I & 0
\end{array}\right) .
\end{gathered}
$$

Возьмем три из четырех матриц Дирака $\frac{1}{\sqrt{2}}(\boldsymbol{\alpha}, \beta)$. Их вторые степени равны $1 / 2$, они взаимно антикоммутируют и действуют на четыре базисных вектора. Таким образом проверяется, что $\mathcal{N}=2^{(N+1) / 2}=4$ для $N=3^{2)}$.

Теперь уже понятно, как надо действовать в случае высших $N$. Чтобы записать $N$ операторов нулевых мод, действующих на состояния, следует использовать алгебру Клиффорда, реализованную в терминах матриц размера $\mathcal{N} \times \mathcal{N}$, и $\mathcal{N}$-компонентные декартовы векторы. При выборе представителей алгебры Клиффорда следует избегать матриц, диагональных в декартовом базисе, поскольку такие элементы отвечают реализациям с нарушенной фермионной четностью. Реализации с сохраненной фермионной четностью содержат соответственно $\mathcal{N}=2^{N / 2}$ и $\mathcal{N}=2^{(N+1) / 2}$ состояний для четного и нечетного числа вихрей. Возникающая алгебра представляет собой алгебру Клиффорда с тем ограничением, что диагональные элементы должны отсутствовать [6].

Интересной открытой проблемой остается вопрос о роли, если она имеет место, реализаций с нарушенной фермионной четностью, которые встречаются в суперсимметричных теориях.

Мы все время надеемся услышать от экспериментаторов новость об обнаружении майорановских частиц. Но кто будет первым: исследователи в области сплошных сред или в области элеменарных частиц?

2) Матрица $\left(\begin{array}{lr}I & 0 \\ 0 & -I\end{array}\right)$ также антикоммутирует с остальными, но ее нельзя использовать, так как она имеет диагональный вид, приводящий к нарушению фермионной четности. 


\section{Список литературы}

[1] R. Jackiw, P. Rossi, Nucl. Phys. B, 190:4 (1981), 681-691.

[2] L. Fu, C. L. Kane, Phys. Rev. Lett., 100:9 (2008), 096407, 4 pp.

[3] C. Chamon, R. Jackiw, Y. Nishida, S.-Y. Pi, L. Santos, Phys. Rev. B, 81:22 (2010), 224515, $10 \mathrm{pp}$.

[4] A. Losev, M. A. Shifman, A. I. Vainshtein, Phys. Lett. B, 522:3-4 (2001), 327-334.

[5] G. W. Semenoff, P. Sodano, Electron. J. Theor. Phys., 3:10 (2006), 157-190.

[6] R. Jackiw, S.-Y. Pi, Phys. Rev. Lett., 85:3 (2012), 033102, 3 pp.

Поступила в редакцию 30.04.2014 